\title{
Criterios para el uso del placebo: Aspectos éticos
}

\section{Criteria for the use of placebo in clinical trials: Ethical considerations}

\author{
Carlos Celedón L1.
}

\section{RESUMEN}

I placebo se podría definir como una terapia médica simulada sin efectos específicos para lo que se está investigando. Se analiza el "efecto placebo", que es el efecto psicológico y fisiológico que produce en el organismo el uso del placebo, a pesar que éstees biológicamente inactivo. En la medicina actual el uso de estudio doble ciego, en los cuales un grupo de pacientes recibe tratamiento con la droga a evaluar y el otro placebo, es el diseño de elección para el estudio de nuevos fármacos. Se analizan las recomendaciones de la declaración de Helsinki sobre el uso de placebo y se indican algunos problemas éticos sobre su uso.

\begin{abstract}
Placebo could be defined as a simulated medical therapy with no specific effects on the subject of investigation. The "placebo effect", i.e., the psychological and physiological effects of placebo on the organism, despite its being biologically inactive, is analyzed. Double-blind studies, in which a group of patients receives treatment with the evaluated drug, while a second group is given placebo, is currently the method of choice when testing new drugs. The Helsinki declaration recommendations on placebo utilization are analyzed, and the ethical implications are discussed.
\end{abstract}

Una investigación puede ser observacional o bien experimental. En el primer caso el investigador solo observa un fenómeno natural, en cambio en el segundo interviene modificando dicho fenómeno o creando un fenómeno artificial. Los ensayos clínicos corresponden a este segundo grupo. Una investigación clínica implica la aplicación en un ser humano de cualquier práctica que afecte su proceso salud-enfermedad con el objetivo de obtener conocimiento sobre dicha práctica. Los objetivos y el diseño de la investigación deben implicar una mejora de las expectativas terapéuticas'1.

Los ensayos clínicos se pueden dividir en libres o abiertos; controlados y controlados aleatorizados. En los primeros, la comparación de la nueva terapia se realiza con experiencias anteriores relativas a la evolución de la enfermedad y al efecto de otros tratamientos; por ello se denominan controles históricos. En los ensayos clínicos controlados se compara el efecto del nuevo tratamiento con la evolución de la persona que no ha recibido terapia alguna o que ha recibido el tratamiento habitual. Comparar con placebo tiene la finalidad de poner en evidencia un determinado efecto terapéutico (generalmente fase II), mientras que comparar con un fármaco activo tiene por finalidad mostrar una diferencia en la relación beneficio/riesgo en una relación clínica concreta (generalmente fase III). Por su parte el ensayo clínico controlado aleatorizado es una forma de establecer el grupo control y que consiste en la selección al azar de quienes van a estar en el grupo control y de quienes van a beneficiarse de la terapia experimental ${ }^{1}$.

\footnotetext{
${ }^{1}$ Médico, Servicio de Otorrinolaringología. Hospital Cínico Universidad de Chile.
} 
日 ensayo clínico controlado (ECC) es la aplicación del método científico a la terapéutica a objeto de distinguir entre terapias útiles y no útiles. Su objetivo es mostrar la eficiencia de un tratamiento por lo que se compara, ej.: una droga nueva, con una droga vigente 0 bien con placebo ${ }^{1-3}$. Los ensayos clínicos controlados que estudian fármacos se diseñan para responder si la droga en cuestión tiene algún efecto farmacológico o no. Algunos diseños usan comparaciones históricas, otros buscan respuestas en relación con la dosis o bien otros, comparaciones con productos inertes (medicación falsa o placebo). Sin embargo la prueba con mayor fuerza científica proviene de la comparación del fármaco de prueba con el placebo $0^{4}$. 日 placebo vendría a ser el "tratamiento patrón" o gold standard contra el cual se comparan los tratamientos de los ensayos clínicos.

日 placebo se podría definir como una intervención diseñada para simular una terapia médica, la cual no tiene efectos específicos para la condición que está siendo aplicada. En otras palabras es un procedimiento que no tiene efectos fisiológicos ni bioquímicos sobre la enfermedad o condición en estudio ${ }^{1,3-5}$.

日 placebo es biológicamente inactivo pero puede producir efectos tanto fisiológicos (disminución del dolor por efecto de la liberación de endorfina) o psicológicos (depresión y dolor). Esto llamado "efecto placebo" es lo que se busca controlar en los ensayos clínicos, a objeto de definir cuáles son los efectos mediados por la intervención en estudio y cuáles son por el "efecto placebo" $1,3-5$. Se ha detectado un efecto terapéutico promedio en alrededor de $30 \%$ en pacientes sometidos a placebo lo cual se explicaría, entre otras, por liberación de endorfinas ${ }^{1,5}$. En los pacientes oncológicos el uso del placebo logra una mejoría de $30 \%$ mitigando el dolor y/o mejorando el ánimo o la esperanza del enfermo ${ }^{6}$. Existen otros factores que pueden influir en estos efectos terapéuticos como son: la fe; confianza en el equipo tratante; esperanza; condicionamiento previo del paciente; elementos simbólicos y culturales ${ }^{1}$. Pérgola citando a Balint nos dice que: " $\boxminus$ médico es medicina, el médico debe tener presente que con su sola presencia tiene un efecto placebo incorporado a su investidura ${ }^{6}$. Por otro lado se puede atribuir equi- vocadamente al efecto placebo situaciones que son propias de la enfermedad, a saber, mejoría espontánea de la enfermedad y/o fluctuaciones de los síntomas ${ }^{5}$. 日 uso del placebo es evidente en algunas enfermedades en que el dolor juega un rol importante y en enfermedades psicosomáticas. Todos estos elementos hay que tenerlos presentes al momento de interpretar los resultados de un "ensayo clínico controlado con placebo (ECCP).

Es importante destacar que el número de consultas de la medicina alternativa supera el número de consultas de los médicos de atención primaria. Habría que investigar cuál es el papel del efecto placebo en estas prácticas médicas. $\mathrm{日} 30 \%$ de los norteamericanos usan terapias alternativas. Se cree que más del $90 \%$ de la medicina alternativa está basada en el efecto placebo ${ }^{7}$.

Los ECCP permiten concluir rápidamente y con menos pacientes si un tratamiento de prueba es mejor que la sustancia inerte o placebo (el cual debe ser idéntico a la droga en estudio) y por otro lado ofrece una respuesta más precisa acerca de los efectos secundarios del fármaco que está siendo probado ${ }^{1,4}$.

En el año 1979 se publica el informe Belmont en el cual se establecen los cuatro principios que deben regir la ética médica: autonomía; beneficencia; no maleficencia y justiciå. Hoy en día está fuera de discusión la íntima relación que existe entre investigación científica y ética. Un ensayo que no está científicamente bien desarrollado deja de ser ético.

En la medicina actual el uso de estudios doble ciego, en los cuales un grupo de pacientes recibe tratamiento con la droga en evaluación y el otro sólo recibe placebo, es el diseño de elección para el estudio de nuevos fármacos ${ }^{9}$.

En la declaración de Helsinki se establece cuándo se puede utilizar el placebo en investigación clínica: "Los posibles riesgos, costos y eficacia de todo procedimiento nuevo deben ser evaluados mediante su comparación con los mejores métodos preventivos, diagnósticos y terapéuticos existentes. Elo no excluye que pueda usarse un placebo, o ningún tratamiento, en estudios para los que no hay procedimientos preventivos, diagnósticos o terapéuticos probados". En el año 2001 se agregó a este punto una nota aclaratoria esta- 
bleciendo que: "Los ensayos con placebo son aceptables únicamente en ciertos casos, incluso si se dispone de una terapia probada pero si se cumplen las siguientes condiciones: a) Quando por razones metodológicas, científicas y apremiantes, su uso es necesario para determinar la eficacia y la seguridad de un método preventivo, diagnóstico o terapéutico; b) Ouando se prueba un método preventivo, diagnóstico o terapéutico para una enfermedad de menos importancia que no implique un riesgo adicional, efectos adversos graves o daño irreversible para los pacientes que reciben el placebo ${ }^{7}$.

日 gran problema ético del uso de placebo en ECC es el de que en virtud del azar a un grupo de pacientes no se les da una terapia existente cuyo beneficio está fuera de duda y no se le advierte al firmar el consentimiento informado de que a un grupo de ellos elegidos al azar, sólo se les dará placebo. Es ético administrar placebo cuando no existe tratamiento establecido para determinada enfermedad o bien cuando la terapia actual tiene demasiados efectos indeseables y se propone una terapia nueva cuyos beneficios hay que probar. En otras palabras se duda de la eficacia de una terapia existente y se quiere probar una nueva comparándola ciegamente con "nada". No debería haber problemas éticos con el uso del placebo en cualquiera circunstancia siempre y cuando se le explique al paciente claramente de qué se trata el uso del placebo, éste lo autorice y lo firme en el consentimiento informado ${ }^{1-4}$.

Existen algunos problemas, al menos dudas desde el punto de vista ético, en cuanto al argumento de que el EOCP permite resultados más rápidos y por consiguiente permite usar ese conocimiento para salvar vidas a pesar de desproteger a los pacientes en el brazo con placebo. Entre estos problemas éticos habrían que mencionar-14:

a) No hay seguridad de que los resultados con un nuevo fármaco sean positivos, de tal manera que sin beneficio para ningún paciente del estudio, se expone a las personas del grupo placebo.

b) Si el resultado es positivo, no garantiza que el medicamento esté disponible de inmediato para beneficiar a otros, como ha ocurrido en Sudáfrica en estudios de Sida. c) Si se desprotege a las personas argumentando los rápidos resultados, entonces los protocolos de investigación no deberían ser rechazados aunque no protejan a los participantes, dado que casi todos ofrecen beneficios para la humanidad.

d) Si se relajan las reglas éticas en aras de lograr resultados rápidos, las personas podrían perder confianza y dejar de participar en los estudios, lo cual dificultaría encontrar voluntarios.

La Administración de Drogas y Alimentos (FDA) establece que un protocolo debe ser aprobado por el centro donde se desarrollará la investigación (esto incluye dos aprobaciones: la del Comité de Etica y la del Comité de Docencia e Investigación) y por un comité de ética independiente. Cada comité tiene una reglamentación sobre el uso del placebo que cumple con los criterios del informe Belmont, de la declaración de Helsinki y de las buenas prácticas clínicas?

Fnalmente habría que recalcar que prácticamente hay unanimidad a nivel mundial, que éticamente se acepta el uso del placebo en investigación clínica, en circunstancias como: a) frente a la ausencia de un tratamiento efectivo; b) cuando el tratamiento con placebo es corto; c) cuando no agrega riesgo no relacionado a su patología de base; y d) cuando el consentimiento informado firmado por el paciente ha sido claro al respecto?.

\section{BIBLIOGRAFÍA}

1. Rodríguez En. y Otomuro D. Eica de los Ensayos Cínicos. En: Lolas F., Quezada A. y Rodríguez Ed. Investigación en Salud: Dimensión Etica $\mathrm{C} \boxplus$, Universidad de Chile. Marzo 2006; 151- 176.

2. VALDIVIESO A. Etica e investigación clínica Bol EsC Med 1998; 27(1): 27-33.

3. Rodrígrez ED. Eección de control en ensayos clínicos, el problema ético del uso del placebo; comentario a las pautas éticas internacionales para la investigación biomédica en seres humanos CIOM 2002. En: Lolas F. y Quezada A. Programa Regio- 
nal de Bioética OPS/OMS- LOM Ediciones Ltda., mayo 2003.

4. Ramírez I, Cabrera C. La bioética y el uso del placebo en ensayos clínicos controlados. Rev Med IMSS2003; 41(1): 65-73.

5. RADA G Placebo y el efecto placebo. http:// escuela.med.puc.cl

6. Ṕ́rgola F, Fustinoni JC. 日 dilema de la terapia del cáncer y la relación oncólogo-paciente. Medicina (Buenos Aires) 1998; 58: 314-8.

7. P T. 日 placebo en la práctica y en la investigación clínica.
8. Informe Belmont. Principios y guías éticos para la protección de los sujetos humanos de investigación. Comisión Nacional para la Protección de los Sujetos Humanos de Investigación Biomédica y del Comportamiento. EEU. 18 de abril de 1979.

9. Papakostas YG, Daras MD. Placebos, placebo effect, and the response to the healing situation: The evolution of a concept. Epilepsia 2001; 42(12): 1614-25.

\footnotetext{
Dirección: Dr. Carlos Celedón Lavín

Luis Thayer Ojeda 0115, O. 705, Providencia

Email: celedon@mi.cl
} 